\title{
Analysis on the KPI and SWOT Analytical Method and the Application on Enterprise Organization Research
}

\author{
Yuanyuan Pan
}

School of Economics and Management of Beijing Jiaotong University, Beijing 100044, China.

\begin{abstract}
This paper is mainly about analysis on the KPI and the SWOT analytical method and the application on enterprise organization research. Enterprise organization is along with the social development and dynamic change. Enterprise based on the hierarchical organizational structure which is gradually rigid and insufficient, has not adapted to the requirement of enterprise development in the information age. Mature application of basic information technology, market competition caused by economic globalization and the diversity of the consumer demand, such as the information age of the enterprise organizational change triggers. Our research integrates the state-of-the-art methodologies and the approaches to propose the novel perspective that is meaningful.
\end{abstract}

Keywords: KPI and SWOT; analytical method; enterprise organization; application; research.

\section{Introduction}

Enterprise organization structure is one of the most important aspects of the enterprise organization and core management research. The evolution of enterprise organization structure on the one hand complies with the requirements of the environmental change; On the other hand also is the need for sustainable development of the enterprise itself. No matter what type of enterprise, its organizational structure is often characterized by a crisscross network the longitudinal hierarchy defines membership and leadership relations between the members of the organization or institution, lateral branch formed on the same level of collaboration between different units or departments [1-2].

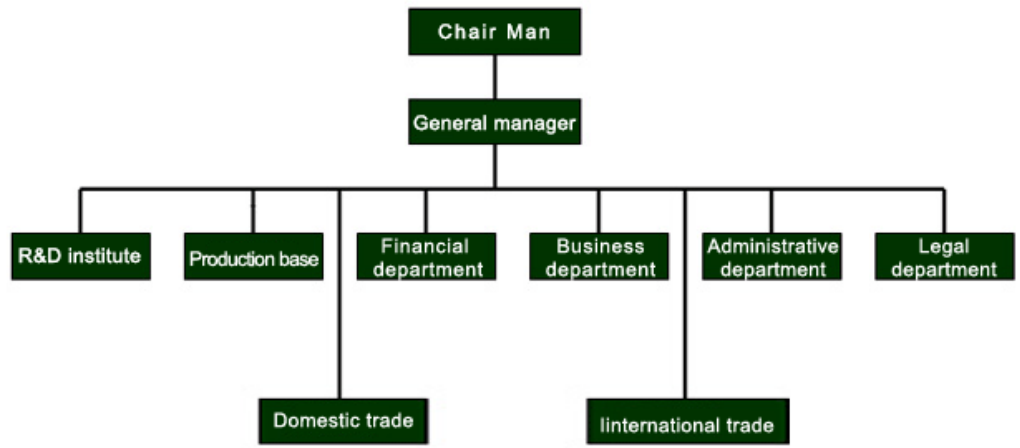

Fig. 1 The General Architecture of the Enterprise Organization

As illustrated in the Fig. 1, we demonstrate the general architecture of enterprise organization. The current open market competition environment and the information into a leading new technology revolution have faced by the enterprise with the competitive situation and competitive landscape where great changes have taken place. Under this basis, we summarize the corresponding challenges for the basic management of the enterprise as follows: (1) Enterprise organization design is based on the existing production guidance, dominated by a seller's market of basic organizational design has neglected the existing market by seller's market into a buyer's market, the enterprise by centering on the products to customer as the center of change. (2) Existing enterprise organizational design ignored coordination between large, medium and small enterprises and their mutual cooperation. (3) Existing mainstream organization design theory is still in a single enterprise organization design problems as the research object, and neglected contemporary enterprise is from the traditional competition gradually towards the joint problems in competition, competition among the enterprises with the problem. (4) Existing enterprise organizational design more emphasize the basic production of a single enterprise internal management structure and management structure. 
In this condition, for the optimization of the enterprise development, we propose the research on the KPI and SWOT analytical method and the application on enterprise organization research. In the following sections, we will discuss the issues in detail.

\section{Our Methodology}

\subsection{Enterprise SWOT Analysis.}

SWOT is an analysis of the important methods for enterprise future development strategy. In this paper, through the analysis of the advantages of internal supply chain financing model runtime environment factors and disadvantaged factors, we found that the pattern of the positive factors and negative factors as affecting the operation of the model by analyzing the external environment of the opportunities and threats factors, which found that affect the business to carry out the favorable and the unfavorable conditions (Fig. 2).

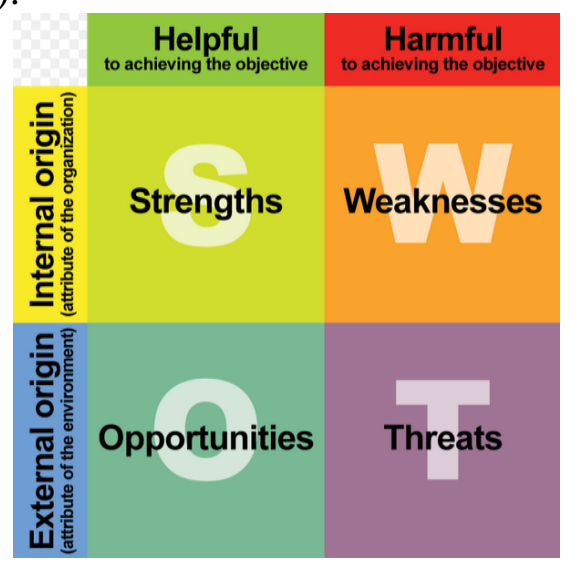

Fig. 2 The Demonstration of the SWOT Analysis Paradigm

As the strategic analysis method, SWOT analysis method can also be divided into four kinds of strategy: SO strategy, ST strategy, WO strategy, WT strategy and their specific relationship as shown in the model. SO strategy: rely on the internal advantage, use external opportunity. ST strategy: rely on the internal advantage, avoid external threats. WO strategy: to overcome the internal weaknesses, using the external opportunity. WT strategy: to overcome the internal weaknesses, avoid external threats. As the demonstration, we summarize the SWOT qualitative analysis of small and the medium-sized enterprise technology innovation as follows: (1) Cost advantage: general small and the medium-sized enterprise flexible mode of operation, management cost is low, small production scale and technology innovation of low cost and small obstacles. (2) Innovation advantage: strong power, speed, as well as aging. Small and medium-sized enterprises are faced with strong external competition, environment selection are great external pressure to enterprise innovation. (3) Structure advantages: clear property rights, interests, information flow, communication is convenient, flexible mechanism and reflect agile. (4) Management weaknesses: small and medium-sized enterprise internal functions are limited, the external environment of low strain capacity, weak ability to obtain external innovation resources, in the process of growth and formed the light weight tactical strategy, decision making at the thinking mode of the feeling experience [3].

\subsection{The KPI Modelling.}

Performance management is to manage both sides agree on goals and how to implement the process. In the whole process of performance management, the design of performance indicators system is very important, work is the key to the performance management which can achieve the desired effect. When designing performance index system, index system of strategic orientation should play the role of traction to go through the layers of core decomposition, enterprise strategic objectives to employees' personal behavior and the department goal, combining the index system to effectively interpret and spread the overall development of the enterprise strategy.

KPI index is to show the enterprise strategic target decision after layer upon layer decomposition of the key, operational performance evaluation index. KPI index system mainly through contributions to individual and group key performance indicator of evaluation basis and establish, through layer 
upon layer decomposition of quantitative system of key performance indicators, the implementation effect of the key activities of the enterprise and its core directly control and measure.

$>\mathrm{KPI}$ is a combination of organization strategy and management method, and to establish the basic index system of the starting point is the organization's strategic objectives, groundwork is to guarantee the realization of the strategic goals, at the same time, through the layers of basic decomposition, the process of the formation of library is a complete comprehensive indicators.

$>$ Set up key performance index system from the organization strategy, based on organization structure, a series of factors such as the scale, nature, the external environment, enacted by the premise of the correct understanding of each selection of key performance indicators are based on the general analysis of the facts.

$>$ For an organization to establish a KPI index system of performance evaluation system is not only enhances the technology for examination and assessment of the company or organization's actual performance, but also is the method of basic strategic management thinking.

Management to set of indicators should be for those who have important effect to achieve company goals, it is temporary and difficult to use KPI to measure the key management and activities. Managing to finish the item is mainly composed of schedule and whether the desired effect to evaluate its strategic significance will assist enhancing the general features of the management procedures.

\subsection{Enterprise Organization Research.}

Reconfigurable organization structure should change from basic strict multi-layer hierarchical structure for the task oriented dynamic hierarchical structure of the flattening. Because hierarchical structure is the inherent attributes of that many artificial system and natural system, therefore, reconfigurable organization structure should reflect in the system of general master-slave constraint relationship.

Enterprise organization structure and the operating mode adapt to general market competition environment, and is suitable to the condition of science and basic technology and at the same time. Organizational change is associated with the development of the science and technology, and the development and popularization of information technology applications for the enterprise mode of production and laid a solid technical foundation enterprise organization model transformation.

Using modern information technology makes the enterprise delivers timely product value chain between the enterprise's business data, improved the work efficiency of the enterprise. The successful use of information systems reduces the operation cost and greatly improves the operating speed. Information among the enterprise groups together, forms a network management system, and rapid responses to changing market demand. The competition in the general market environment of the information age, the scale of the enterprise is no longer as decision success or failure of enterprise management decision power. On the contrary, the enterprise can quickly adapt to the changing market needs, as key elements decide the success or failure of the enterprise.

$>$ Process reengineering. Refactoring can be divided into following stages: process modeling, process analysis and reengineering. Process modeling using formalized language to describe the target, content, activity decomposition. Process analysis and reengineering is the hierarchical decomposition of the event, to establish activity function tree.

$>$ Enterprise organization between refactoring. Enterprise organization between reconstructions refers to a number of enterprises established long-term and short-term group or union, in order to fully meet customer needs and maximize benefits. Enterprise organization between refactor also can be further divided into static organization between enterprise and dynamic organization reconstruction [4].

$>$ The influential factors of general organization reconstruction. Enterprise development strategy determines enterprise development goals that market orientation and mode of operation, direct effects on enterprise organization reconstruction. In the changing market 
environment in the actual operating conditions and enterprise development strategy formulation goal deviation, enterprises need to refactor.

\subsection{The Enterprise Management.}

Overall systematic modern enterprise management innovation, this feature is the result of the systemic of the enterprise as it is to point to managers looking for key points in enterprise management innovation, and evaluation of management innovation achievements according to enterprise's system. Enterprise is a complex system the elements in the system connect with each other and interact, when the system within certain or some elements are in a bad state, there will be affected by other factors, at the same time, the enterprise system as a whole will also be in the bad state. Ecological enterprise is based on ecological economics and ecology system is efficient, harmonious, optimization theory with the integrated use of the ecological engineering methods and everything is advantageous to the enterprise for sustainable development of the modern science and technology, design, and reforming the process flow of the enterprise, organization reasonable cycle of enterprise internal production process.

Enterprise management innovation is not only reflected on inheritance of scientific management and perfect, but also to the management concept, the management strategy, organizational structure, management methods and systemic adjustment of enterprise culture. By improving the management innovation, enterprises will get sustained competitiveness, and it has a big impact on organizational performance, to ensure that enterprises in a dynamic environment forward steadily and durability. Management innovation on the role of technology innovation is embodied in every process of the technological innovation has the management innovation that management innovation provides the organization guarantee for technological innovation, create the necessary management environment and conditions. Management innovation ability from the technology innovation ability that can fully effective research enterprise management innovation ability, and can explain the relationship between management innovation and technological innovation well.

\section{Conclusion}

In this paper, we conduct analysis on the KPI and the SWOT analytical method and the application on enterprise organization research. Enterprise management innovation is important according to the objective requirements of the production, business operation and conditions of the development of science and technology. With the development of our economy, basic market economy is gradually established and needs enhancement of the internationalization. In order to make enterprises survive and develop in the increasingly fierce market competition, it is necessary to constantly improve the level of modern management to enhance the ability of management innovation. In the further research, we will enhance the contemporary methodology with more corresponding theories.

\section{References}

[1] Imperatori, Barbara, and Dino Cataldo Ruta. "Designing a social enterprise: Organization configuration and social stakeholders' work involvement." Social Enterprise Journal 11.3 (2015).

[2] Jing, Zhu, et al. "The Research on Enterprise Organization Constructer Reforming Based on Internal Marketing." (2013).

[3] Choo, Bennie Seck-Yong, and Jenson Chong-Leng Goh. "Pragmatic adaptation of the ISO 31000: 2009 enterprise risk management framework in a high-tech organization using Six Sigma." International Journal of Accounting \& Information Management 23.4 (2015): 364-382.

[4] Song, Jiekun, and Yu Zhang. "Study on the partners selection and investment allocation models of enterprise cooperative competitive intelligence." Service Systems and Service Management (ICSSSM), 2012 9th International Conference on. IEEE, 2012. 